\title{
Malignant peripheral nerve sheath tumour (MPNST) of thorax: a rare soft tissue sarcoma in a patient with neurofibromatosis type 1
}

\author{
Thanancheyan $\mathbf{S}^{1}$, Muthulingam $\mathbf{A}^{1}$, Selvaratnam $\mathbf{N}^{1}$ \\ Journal of the Ceylon College of Physicians, 2021, 52, 40-44
}

\begin{abstract}
Neurofibromatosis is a genetic disorder with multisystem involvement. Malignant peripheral nerve sheath tumours (MPNST) are one of the soft tissue sarcomas associated with neurofibromatosis type 1 (NF-1). These tumours are relatively rare and mostly occur in proximal portions of the upper and lower extremities and the trunk. MPNST of the thoracic cavity has rarely been reported. This case report describes a woman with NF-1 who presented with progressive dyspnoea and was found to have a mass in the thoracic cavity. She underwent partial resection and was found to have MPNST. Intrathoracic MPNST have a bad prognosis often needing neoadjuvant chemotherapy or chemoradiation pre/ post resection.
\end{abstract}

Key words: neurofibromatosis, soft tissue sarcomas, malignant peripheral nerve sheath tumours

\section{Introduction}

Neurofibromatosis (NF) is a genetic disorder with three distinguishable forms named NF type 1(NF-1), type 2 (NF-2), and schwannomatosis. Among these three types, NF-1 is the most common. ${ }^{1} \mathrm{NF}-1$ occurs due to mutation in NF 1 gene located on chromosome 17 and it could be either spontaneous or inherited with autosomal dominant pattern. ${ }^{2}$ NF 1 gene encodes neurofibromin; a protein that participates in cell proliferation and differentiation and functions as a tumour suppressor. NF-1 has an estimated incidence of 1 in 3000 to 3500 individuals. ${ }^{2,3}$

It has been observed that the individuals with NF-1 have a high risk to develop tumours, both benign and malignant, during their lifetime. Neurofibromas (peripheral, cutaneous, plexiform and nodular), which are benign, are the most common tumours. Other tumours include optic pathway gliomas (OPG), low grade astrocytomas, brain stem gliomas, soft tissue sarcomas, some types of leukaemia and phaeochromocytoma. Soft tissue sarcomas associated with NF-1 comprise malignant peripheral nerve sheath tumours (MPNST), rhabdomyosarcoma, gastrointestinal stromal tumours and glomus tumours. ${ }^{1}$

\section{Clinical presentation}

A 29-year-old woman, known patient with neurofibromatosis, was admitted to Teaching Hospital, Jaffna with complaints of dry cough and shortness of breath over one-week duration. She had pleuritic type of chest pain and palpitations. She denied history of fever. There was no contact history of tuberculosis or SARS-Co-V2 infection.

On examination, she had numerous neurofibromas. She was tachypnoeic, afebrile and not pale or cyanosed. Oxygen saturation $\left(\mathrm{SpO}_{2}\right)$ on room air was $98 \%$. Blood pressure was $121 / 73 \mathrm{mmHg}$ with pulse rate of $140 \mathrm{bpm}$. Auscultation of lungs revealed bilateral rhonchi and reduced breath sounds in right middle and lower zones. Chest radiography showed a mass lesion in the right lung field. Echocardiography was normal.

${ }^{1}$ Teaching Hospital, Jaffna, Sri Lanka.

Correspondence: ST, e-mail: sanchai1990@gmail.com

iD https://orcid.org/0000-0001-6906-385X

Received 27 February 2021, accepted 15 April 2021.

This is an open-access article distributed under the terms of the Creative Commons Attribution License, which permits unrestricted use, distribution, and reproduction in any medium, provided the original author and source are credited. 
Contrast enhanced computerized tomography (CECT) of chest showed a large $(16.3 \times 12.3 \times 9.4 \mathrm{~cm})$ heterogeneously enhancing extra pulmonary mass with intact tissue planes in the right hemi thorax consistent with plexiform neurofibromas. Right lung was partially collapsed. There was a similar smaller lesion in the left hemi thorax. There were no pathological mediastinal lymph nodes (Figure 1 ). White blood cells $12.3 \times 10^{9} / \mathrm{L}$ (4-11), haemoglobin $10.1 \mathrm{~g} / \mathrm{dL}$ (11.0-16.0) platelets 516 10\% (150-450) and ESR $25 \mathrm{~mm} / 1 \mathrm{st}$ hour (0-30) and CRP was $68 \mathrm{mg} / \mathrm{L}(0-3)$. Rest of the investigations were normal and retroviral and TB screenings were negative.

She was commenced on intravenous ceftriaxone $1 \mathrm{~g}$ b.i.d, oral clarithromycin $500 \mathrm{mg}$ b.i.d and intravenous dexamethasone $8 \mathrm{mg}$ t.i.d. Her oxygen saturation and haemodynamic parameters were closely monitored. She was transferred to National Hospital for Respiratory Diseases (NHRD), Welisara for urgent surgical intervention.
At NHRD, she underwent clamshell thoracotomy and was found to have a haemorrhagic pleural effusion and tumour in apical and posterior aspect of chest compressing the right lung with infiltration into lower superior vena cava. Deposits were noted in pericardium and upper lobe of left lung. Debulking of right sided tumour and resection of pericardial deposits were done. Deposits in the left lung were left untouched as it required a complicated lobectomy. Her post-operative recovery was uneventful. Pleural fluid culture was negative for bacteria. Histological examination of the resected right thoracic mass was consistent with a high-grade sarcoma (Figure 2). Tumour cells showed positivity for S-100 protein with around $50 \%$ positivity for Ki 67 protein. Immunohistochemistry was negative for smooth muscle actin (SMA) and CD 34 proteins. Pericardial deposits did not show evidence of metastatic sarcoma. She was subsequently referred to the oncology team for further management.

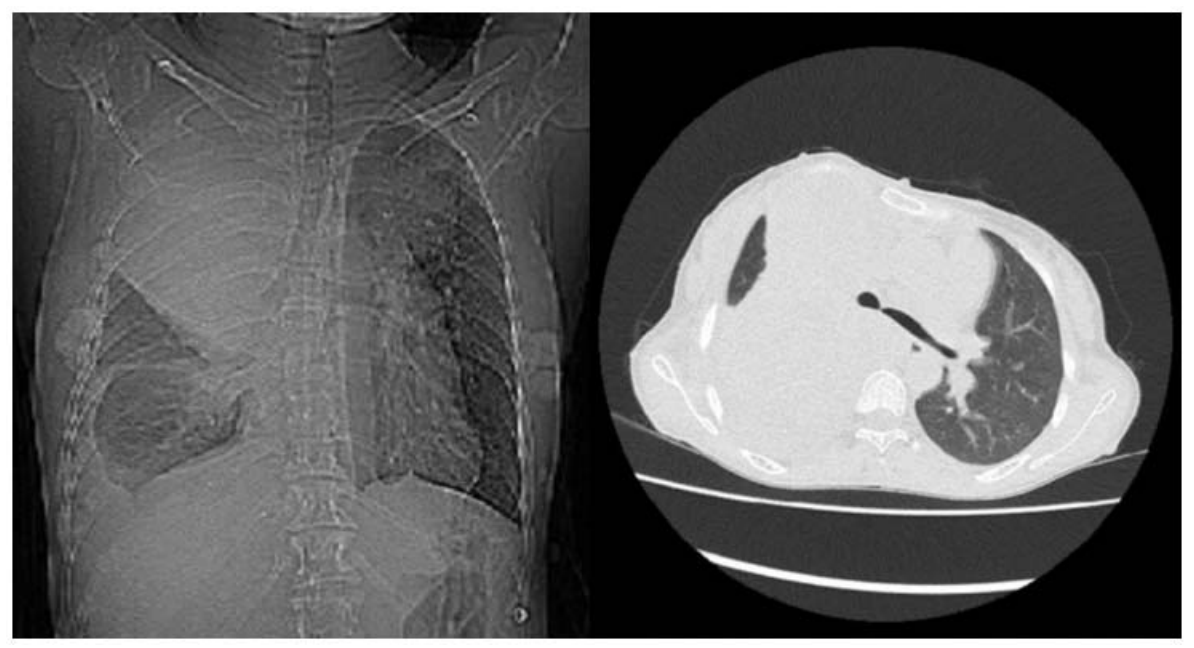

Figure 1. Plain chest CT scan mediastinal window (left) and CECT axial view (right) showing mass lesion in right hemithorax.

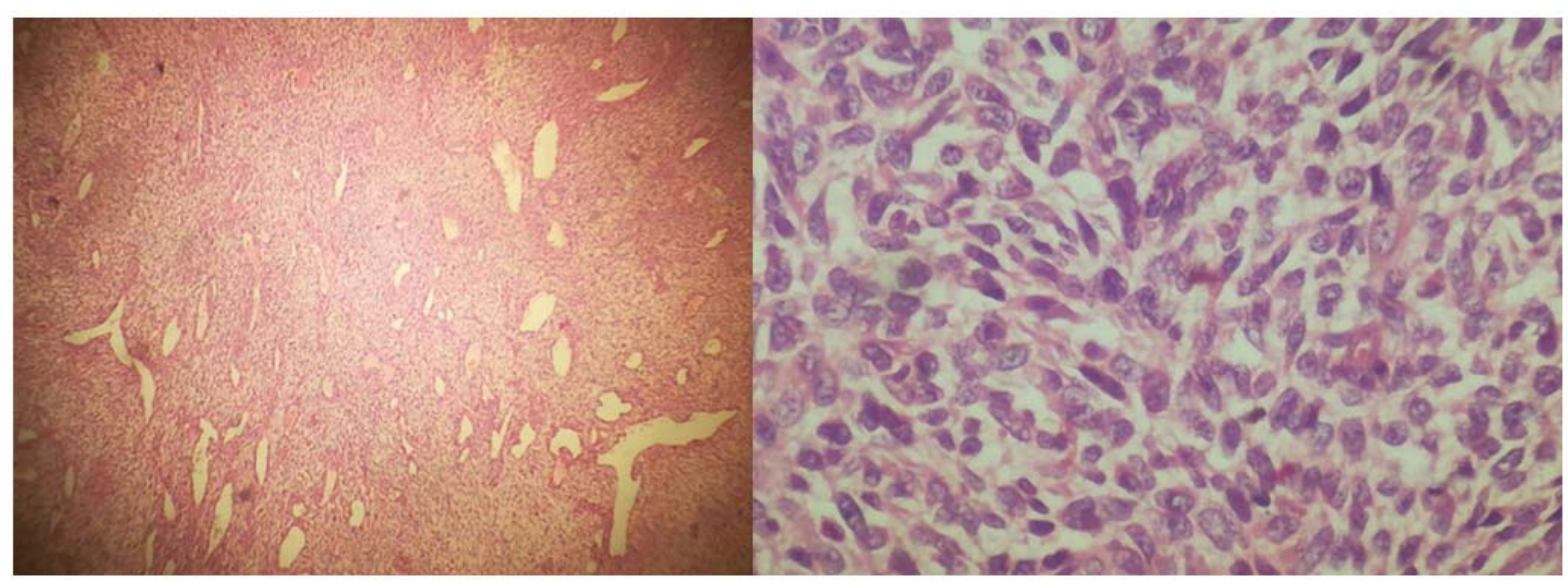

Figure 2. Light microscopy of the resected tumour. Low power magnification (x4) shows marbled appearance (left). High power magnification (x40) shows elongated nuclei and bipolar processes (right) 


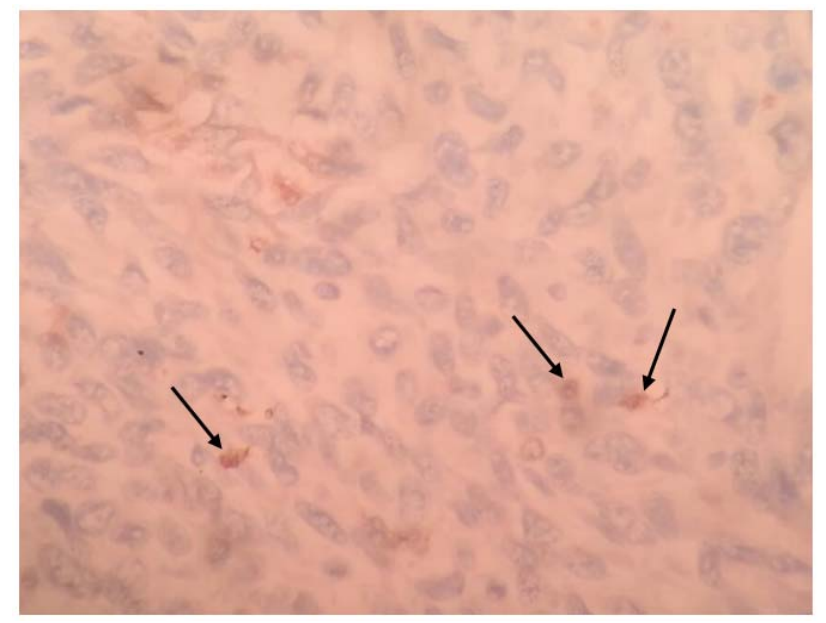

Figure 3. High power magnification (x40) of immunohistochemistry staining shows positivity for S-100 (arrows)
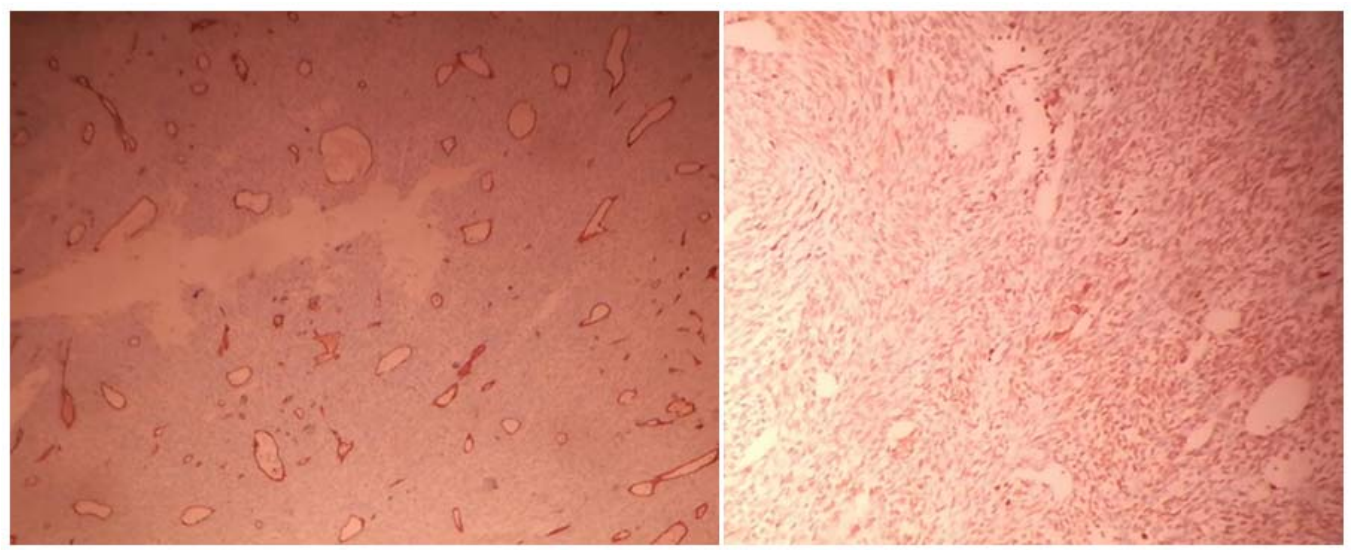

Figure 4. Tumour cells stained negative for CD34 (left) (x4 magnification) and SMA (right) (x10 magnification) in immunohistochemistry. The enhanced vascular staining of CD34 is due to the endothelial cells which is a normal finding.

\section{Discussion}

MPNST is a rare soft tissue malignancy and around $50 \%$ of cases are found in patients with NF-1. It often occurs in adulthood. ${ }^{4,5}$ About $3 \%$ to $10 \%$ of patients with NF-1 develop a MPNST. ${ }^{5}$ Regarded as devastating tumours, most of the MPNSTs in NF-1 end up in poor outcome. ${ }^{4,6}$ The incidence of MPNST is 1 in 100,000 persons. MPNSTs appear to occur much earlier in patients with NF-1 when compared to the normal population. ${ }^{6}$ Although MPNSTs can occur anywhere in the body, they are usually found in extremities and the trunk. MPNSTs are very rarely found in the thoracic cavity as in this case..$^{6,7.8}$ In a case series, intrathoracic MPNSTs were found at the mediastinum (anterior, middle and posterior), in the chest wall, in the lung, and in the paraspinal region. ${ }^{9}$
Patients with thoracic cavity MPNSTs present with, pleuritic chest pain, back pain, painful chest wall lumps, arm pain, and dyspnoea. Asymptomatic presentation also has been documented. ${ }^{9}$

MPNSTs usually arise from pre-existing plexiform neurofibromas or perineuriomas, and exhibit a 20-fold increased risk with the former. MPNSTs can arise de novo from normal nerves as well. ${ }^{6,10}$ Clinically rapid changes in tumour size, onset of significant pain and progression of neurologic deficit are indicative of malignant transformation. ${ }^{6}$

Imaging is not reliable to differentiate malignant and benign tumours, although some features such as tumour size $>5 \mathrm{~cm}$, tumour invasion of fat planes, 
presence of perilesional oedema and poorly defined tumour margins are strongly suggestive of the former. In this case, except the size of the mass, there were no typical features favouring a malignant transformation. In these circumstances fluorodeoxyglucosepositron emission tomography (FDG-PET) could be useful in detecting a malignant tumour but its use is hindered by low specificity. ${ }^{11}$

Definitive diagnosis is made by demonstrating the histology of tumour biopsy. ${ }^{6}$ Histologic findings are of a wide range and usually include cells with elongated nuclei and bipolar processes resembling Schwann cells, presence of a fascicle formation, mitoses, necrosis and extreme nuclear anaplasia. ${ }^{10}$ In low power magnification, these tissues show marbled appearance due to alternating hypocellular and hypercellular areas with perivascular accentuation. Glandular elements are usually absent. ${ }^{12}$

S-100, CD56 and protein gene product 9.5 (PGP 9.5) are considered sensitive markers for MPNST. Though S-100 is generally considered as the best marker for MPNST, it has limited sensitivity of $50-90 \%$ rendering their diagnostic utility in question while CD56 and PGP 9.5 lack specificity. Nestin, which is more sensitive, could be useful in the diagnosis when used in combination with above mentioned tumour markers. ${ }^{13}$

Staging and treatment of MPNSTs are similar to malignant soft tissue sarcomas. For MPNSTs arising in extremities, which are the commonest sites, surgical resection with a sufficient wide margin is the preferred treatment. Adjunctive radiotherapy, either pre-operative or post-operative, can help to salvage the limb and improve local control to achieve wide excision margins. Nerve grafting is not appropriate. At other sites, wide excision is usually impossible due to the complexity of local anatomy. ${ }^{6}$ As with other soft tissue sarcomas, chemotherapy is mainly reserved for unresectable tumours and metastatic MPNSTs. ${ }^{14}$

Surgery has been the treatment of choice whenever possible in case of intrathoracic MPNSTs. Neoadjuvant chemotherapy or chemoradiation is given in patients with a large tumour mass. Patients with nonradical resection receive adjuvant chemotherapy. ${ }^{9}$ This patient's tumour was not completely resectable and she underwent debulking surgery followed by chemotherapy. Neoadjuvant chemotherapy/chemoradiation was not considered in this patient as there was no convincing evidence for malignant transformation before surgery.

Prognosis of MPNSTs are poor despite aggressive surgical and radiation treatment with a 5-year survival rate ranging from $34 \%-64 \%$. Poor prognostic factors are tumours $>5 \mathrm{~cm}$, higher tumour grade, association with NF1, older age, distant metastases at the time of diagnosis, and inability to achieve tumour-free margins (Figure 3). ${ }^{6,15}$

\section{Conclusion}

MPNST is a complication associated with NF-1. Albeit very rare, these tumours carry bad prognosis. Management, which mainly consist of surgical resection, is often complicated by the anatomical site of the tumour. Intra thoracic MPNSTs are much rarer and often need neoadjuvant chemotherapy or chemoradiation prior to surgery. MPNSTs which are not suitable for resection need chemotherapy.

\section{Acknowledgement}

We thank the cardiothoracic surgical team of NHRD who operated the tumour and to the Department of Histopathology, NHRD for providing histological diagnosis.

\section{References}

1. Korf BR, Lobbous M, Metrock LK. Neurofibromatosis type 1 (NF1): Pathogenesis, clinical features, and diagnosis. In A. F. Eichler (Ed.), UpToDate. Retrieved January 28, 2021, from https://www.uptodate.com/contents/neurofibromatosistype-1-nf1-pathogenesis-clinical-features-and-diagnosis

2. Boyd KP, Korf BR, Theos A. Neurofibromatosis type 1. J Am Acad Dermatol. 2009; 61(1):1-16. https://doi.org/10.1016/ j.jaad.2008.12.051

3. Antônio JR, Goloni-Bertollo EM, Trídico LA. Neurofibromatosis: chronological history and current issues. An Bras Dermatol. 2013; 88(3): 329-43. https://doi.org/10.1590/ abd1806-4841.20132125

4. Yoon JH, Lee H-S, Chun JI, Park S-Y, Park HJ, Park B-K. Huge Intrathoracic Malignant Peripheral Nerve Sheath Tumor in an Adolescent with Neurofibromatosis Type 1. Case Reports in Pediatrics 2014; 2014: 951252.

5. LeGallo RD, Wick MR. Soft Tissue. In P. Gattuso, V. B. Reddy, O. David, D. J. Spitz, \& M. H. Haber (Eds.), Differential Diagnosis in Surgical Pathology, Chapter 17, 2015. 3rd ed., pp. 870-925. Philadelphia: Saunders, an imprint of Elsevier Inc. Retrieved January 28, 2021, from https://www. clinicalkey.com/\#!/content/book/3-s2.0 B9781455770137000176

6. Gilchrist JM, Donahue JE. (2020). Peripheral nerve tumors. In A. F. Eichler (Ed.), UpToDate. Retrieved January 28, 2021, from https://www.uptodate.com/contents/peripheral-nervetumors

7. Shanmugasundaram S, Sachdeva AK, Penumadu PS. (2018). Malignant peripheral nerve sheath tumor of the thorax: Unusual location of a rare tumor. Journal of Cancer Research and Therapeutics 14(5), 1145-8. https://doi.org/10.4103/ 0973-1482.203595 
8. Streit S, Izzo C, Najjar N. (2020, October 01). Malignant peripheral nerve sheath tumour spread to the thoracic cavity causing obstructive shock. Chest 2020; 158(4), A1648. https://doi.org/10.1016/j.chest.2020.08.1484

9. Kamran SC, Shinagare AB, Howard SAH, et al. Intrathoracic malignant peripheral nerve sheath tumors: imaging features and implications for management. Radiol Oncol. 2013; 47(3): 230-8. https://doi.org/10.2478/raon-2013-0047

10. Frosch MP. The Nervous System. In V. Kumar, A. K. Abbas, N. Fausto, \& R. N. Mitchell, Robbins Basic Pathology 2007. 8th ed., p. 900. Elsevier. Retrieved February 04, 2021.

11. Bredella MA, Torriani M, Hornicek F, Ouellette HA, Plamer WE, Williams Z, Fischman AJ, Plotkin SR. Value of PET in the Assessment of Patients with Neurofibromatosis Type 1. American Journal of Roentgenology 2007; 189(4): 928-35. https://doi.org/10.2214/AJR.07.2060
12. Kao E, Mantilla JG. Malignant peripheral nerve sheath tumor (MPNST). Pathology Outlines.com website. https:// www.pathologyoutlines.com/topic/softtissuempnst.html. Accessed February 3rd, 2021.

13. Guo A, Liu A, Wei L, Song X. Malignant peripheral nerve sheath tumors: differentiation patterns and immunohistochemical features - a mini-review and our new findings. J Cancer 2012; 3: 303-9. https://doi.org/10.7150/jca.4179

14. Wu LMN, Lu QR. Therapeutic targets for malignant peripheral nerve sheath tumors. Future Neurology 2019; 14(1): FNL7. https://doi.org/10.2217/fnl-2018-0026

15. Ducatman BS, Scheithauer BW, Piepgras DG, Reiman HM, Ilstrup DM. Malignant peripheral nerve sheath tumors. A clinicopathologic study of 120 cases. Cancer 1986; 57(10): 2006-21. 